\title{
Heterozygous clones in Streptomyces coelicolor
}

\author{
By D. A. HOPWOOD* \\ Botany School, University of Cambridge \\ AND G. SERMONTI AND ISABELLA SPADA-SERMONTI \\ Research Unit for Microbial Genetics, \\ Istituto Superiore di Sanità, Rome, Italy
}

(Received 10 May 1962)

\begin{abstract}
SUMMARY
Certain of the colonies that arise when spores from a mixed culture of two complementary auxotrophic strains of Streptomyces coelicolor $\mathbf{A 3}(\mathbf{2})$ are plated on a selective medium give rise to a mixture of spores with parental and recombinant genotypes. These heterogeneous colonies (heteroclones) probably develop from spores containing heterozygous nuclei which segregate during the development of the colonies. The heterozygous nuclei probably undergo a number of equational divisions before segregating. The markers in two linkage groups segregate independently. Large imbalances in the allele ratios and in the frequencies of complementary genotypes have been attributed to terminal chromosome deletions which affect both chromosome pairs. Absence (or homozygosity of the markers) of one whole chromosome is observed very often in heteroclones selected on media which make one chromosome dispensable. The heteroclones provide an efficient system for studying the genetics of the organism without recourse to selective methods.
\end{abstract}

\section{INTRODUCTION}

The actinomycete Streptomyces coelicolor is a filamentous organism which grows in the form of a mycelium and produces regular chains of uninucleate haploid spores as the terminal stage in the life-cycle. There is no morphological evidence of a sexual cycle (Hopwood, 1960), but genetic recombination occurs; it was discovered independently by Sermonti \& Spada-Sermonti (1955) and by Hopwood (1957). When two strains of the organism, each having one or more biochemical deficiencies, are grown together in mixed culture for a few days and spores then harvested and plated on a medium lacking at least one of the growth-factors required by each parent strain, 1 in $10^{2}$ to $10^{5}$ of the plated spores gives rise to a colony. These colonies are of two kinds: haploid recombinants and segregating heterozygotes. The relative proportions of the two kinds of colonies vary according to the particular combination of nutritional requirements against which the medium selects. The haploid recombinant colonies are homogeneous, breeding true on subculture. The relative frequencies of the different genotypes of recombinants can be used to determine the linkage relations of the markers, and two linkage groups each represented by half a dozen loci were recognized by Hopwood (1959). The segre-

* Present address : Department of Genetics, University of Glasgow. 
gating heterozygous colonies, on the other hand, contain in their spores a mixture of different genotypes, and these colonies have therefore been called heteroclones (Sermonti, Mancinelli \& Spada-Sermonti, 1960). The evidence suggests that they arise from single spores (or, more precisely, single 'plating-units') each containing a heterozygous nucleus which allows them to grow on the selective medium.

The heteroclones make up a significant proportion of the total colonies which arise on a selective medium only when the nutritional requirements (at least one in each parental strain) which the medium does not satisfy are determined by closely linked mutants. Under these conditions, only a small proportion of the total haploid recombinants in the plated spore suspension are able to give rise to colonies, while the number of heterozygotes that can grow is unaffected by the stringency of selection. When the two markers selected against are about five recombination units apart, heteroclones represent about $10 \%$ of the total colonies, and the proportion rises to nearly $100 \%$ when the selected markers are less than one unit apart.

The heterozygous nuclei segregate during the growth of the heteroclones, and nearly all the spores to which they give rise are haploid. Since the heterozygous nuclei contain two closely linked nutritional mutants, one in each parental genome, the great majority of the haploid spores have one or other growth requirement and are unable to grow on the selective medium. This provides a ready means of recognizing the heteroclones. Replica plates are prepared from the original selective plates, on a medium of the same composition, by using a velvet pad (Lederberg \& Lederberg, 1952); the homogeneous recombinants are perpetuated on the replica plates, while the heteroclones are not (Hopwood \& Sermonti, 1962).

The interest of the heteroclones of Streptomyces coelicolor lies primarily in the fact that in them the heterozygous condition of the nucleus is prolonged enough for its segregation to be studied. In eubacteria, on the other hand, the heterozygous condition produced by transfer of genetic material from a donor to an acceptor cell is generally very short-lived. When transfer is by Hfr-mediated conjugation (Hayes, 1960), transformation (Ephrussi-Taylor, 1960) or 'general' transduction (Hartman \& Goodgal, 1959), the transferred fragment appears not to reproduce itself, or to do so to a very limited extent, before its incorporation in a recombinant genome. Thus the acceptor cell does not produce a clone of heterozygous cells. Only when genetic transfer is mediated by episomes (Jacob \& Wollman, 1958) do clones of heterozygous cells occur regularly, as in 'special' transduction (Morse, Lederberg \& Lederberg, 1956a) and in 'sexduction' (Jacob \& Adelberg, 1959), where attachment of a fragment of bacterial chromosome to an episome apparently enables it to reproduce with the acceptor cell.

The persistent heterozygotes of Escherichia coli, discovered by Lederberg (1949), represent an exception. In the presence of a mutant gene (Het), the zygotes were able to undergo several divisions before segregation took place. These heterozygotes have contributed little to the picture of the genetic system of $E$. coli; other lines of investigation have been exploited instead (Wollman, Jacob \& Hayes, 1956), and no recent work on the heterozygotes appears to have been published. The heterozygotes of $E$. coli seem to have some features in common with those of Streptomyces coelicolor. However, in $S$. coelicolor they have not only facilitated the formal genetic analysis of the organism, but have also provided some information on the genetic 
system involved in the formation of recombinants. In this paper we shall describe the analysis of the heterozygotes and discuss the information about the genetic system that can be obtained from them.

\section{METHODS}

Mutant strains. All the auxotrophic and streptomycin-resistant mutants used in this work have been derived from the same wild-type strain of Streptomyces coelicolor (Hopwood, 1959). The origins, characteristics and linkage relations of the various mutants were recently summarized by Hopwood \& Sermonti (1962). The markers mentioned in the present paper are located in the two linkage groups as follows, the distances being expressed as \% recombination as deduced from the analysis of heteroclones:

I

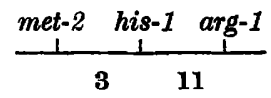

II

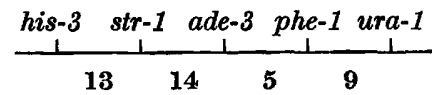

The symbols for the markers are as follows: ade, arg, his, met, phe, ura $=$ requirement for adenine, arginine, histidine, methionine, phenylalanine or uracil, respectively; str $=$ resistance to streptomycin.

Media. For details of the complete and minimal media and of the supplements added to minimal medium, see Hopwood \& Sermonti (1962).

Preparation of mixed cultures and plating on selective media. Two pairs of closely linked nutritional markers were used for the selection of heteroclones: either met- 2 and his- 1 in linkage group I, or ade-3 and phe-1 in linkage group II. Various other markers were incorporated in the parent strains, depending on the scope of the experiment. Inocula of the two parent strains were mixed together on a slope of complete medium. After incubation of the culture for 3-5 days at $30^{\circ}$, a suspension of spores was prepared in sterile water, filtered through cotton wool to remove large mycelial fragments, and spread on plates of selective medium at a density of about $10^{5}$ to $10^{6}$ spores per plate. The selective medium lacked the growth factors corresponding to the pair of closely linked markers, and might or might not contain growth factors corresponding to other markers.

Recognition and study of the heteroclones. When the recombinant colonies were sporulating on the selective medium (about 4 days), replica plates were prepared on a medium of the same composition. Those colonies which did not give growth on the replica plates after incubation for 2 days were identified as heteroclones. The heteroclones were usually, though not invariably, smaller than the homogeneous recombinant colonies. Spores were isolated from a heteroclone in a loopful of water and plated on complete medium at suitable dilutions to obtain isolated segregant colonies. Sometimes spores were also plated on selective media for the recovery of second-order heteroclones. After growth for 3 days the segregant colonies were inoculated to master plates of complete medium, and were characterized by replicating to plates of diagnostic media (Hopwood \& Sermonti, 1962). 


\section{RESULTS}

\section{Quantitative analysis of heteroclones}

Table 1 shows the genotypes of the segregants obtained from a single heteroclone that was isolated on unsupplemented minimal medium. Six markers were segregating, two in linkage group I and four in linkage group II. In a sample of 166 segregants, 23 of the 64 possible genotypes were found, six of thembeing represented only once. This indicates that many more genotypes would have been recovered by isolating a larger sample of segregants. The results are presented as a contingency table in which the relative frequencies of the four combinations of markers in linkage group I are shown in association with each combination of markers in group II. There is no evidence of heterogeneity in the table, since $G$ calculated by

\section{Table 1. Numbers of segregants of each genotype in a heteroclone}

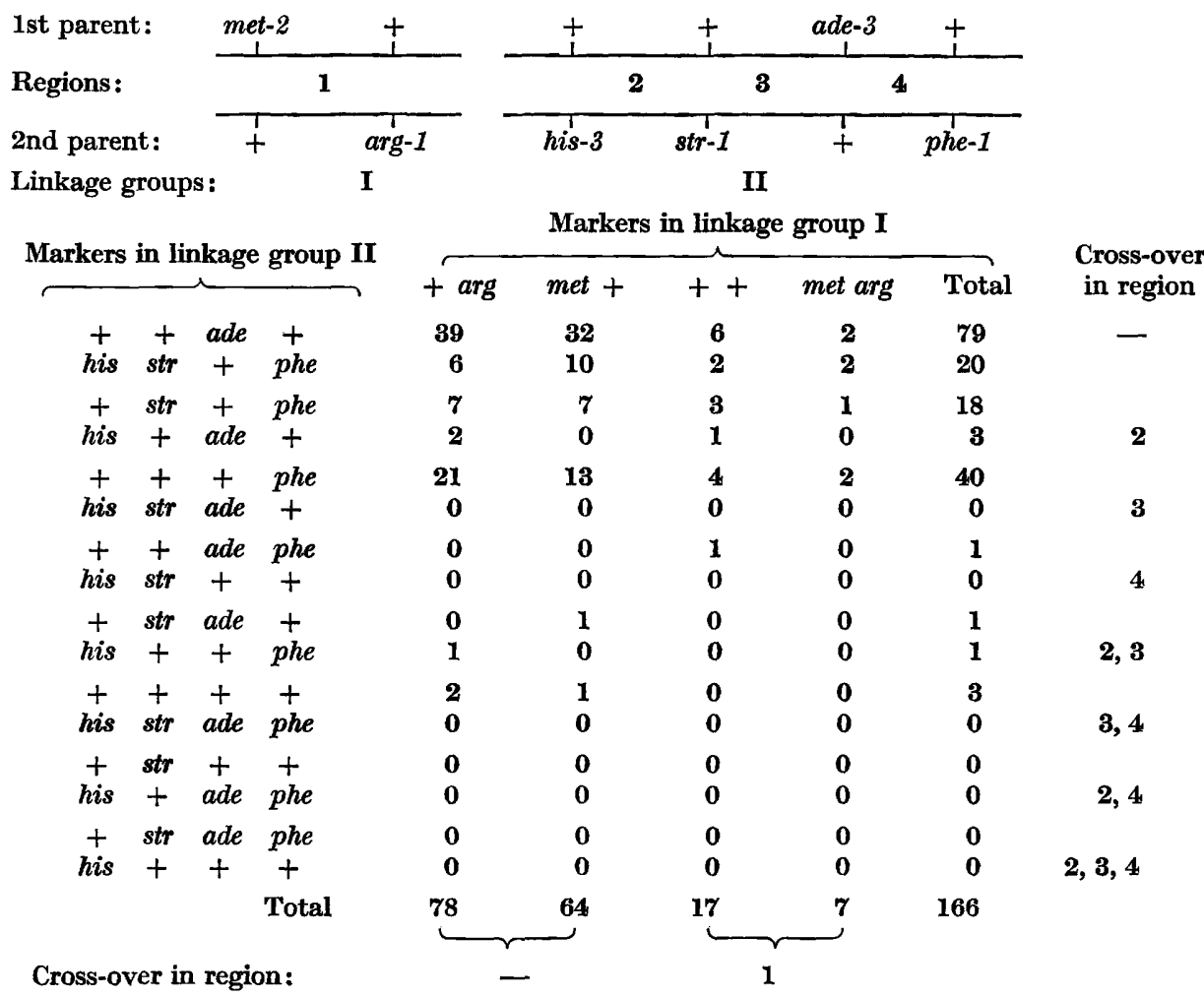

Of the 166 segregants, 157 (those in rows $1,2,3$ and 5 ) have been used to test the independence of marker combinations in the two linkage groups (see text).

the method of Woolf (1957) is equal to 6.09, with 9 degrees of freedom; this corresponds to a probability of $\mathbf{0 . 7 5}$. Thus marker combinations of the two linkage groups are associated at random. This indicates the absence, in this heteroclone, of preferential associations of unlinked markers such as result in some heteroclones from contamination by an extraneous clone, or from the presence of a fast-growing sector originated within the heteroclone. The independence test is used routinely 
to recognize heteroclones in which segregations are disturbed by such effects, and only those segregations which show no preferential combinations of unlinked markers are analysed quantitatively. In Table 1, the proportions of the various pairs of complementary genotypes are in general those predictable from the linkage relations of the markers. However, complementary genotypes are not equally numerous, those containing the allele his-3- being in the minority. Such inequalities in the frequencies of complementary genotypes are characteristic of the heteroclones; their interpretation and their effects on the estimation of linkage will now be considered.

Table 2. Segregation of markers in linkage group II in a heteroclone

\begin{tabular}{|c|c|c|c|c|}
\hline \multirow{2}{*}{$\begin{array}{l}\text { Constitution of the } \\
\text { heterogenote:* }\end{array}$} & + & \pm & \multicolumn{2}{|l|}{ ura-1 } \\
\hline & & & 2 & $y$ \\
\hline \multirow{3}{*}{ Allele ratios: } & str-1 & ade-3 & + & \\
\hline & 88 & 98 & 103 & \\
\hline & $\overline{43}$ & $\overrightarrow{\mathbf{3 3}}$ & $\overline{28}$ & \\
\hline
\end{tabular}

(a) Numbers of segregants of each genotype

$\begin{array}{ccc}\begin{array}{c}\text { Genotypes of } \\ \text { segregants }\end{array} & \begin{array}{c}\text { Observed } \\ \text { numbers }\end{array} & \begin{array}{c}\text { Cross-over } \\ \text { in regions }\end{array} \\ +\quad+\text { ura } & 77 & - \\ \text { str ade }+ & 19 & y \\ \text { str }+ \text { ura } & 16 & 1 \\ + \text { ade }+ & 4 & 1, y \\ \text { str ade ura } & 8 & 2 \\ +\quad+\quad+ & 5 & \mathbf{2 , y} \\ + \text { ade ura } & 2 & 1,2 \\ \text { str }+ \text { + } & \mathbf{0} & \mathbf{1 , 2 , y}\end{array}$

(b) Recombination between pairs of loci

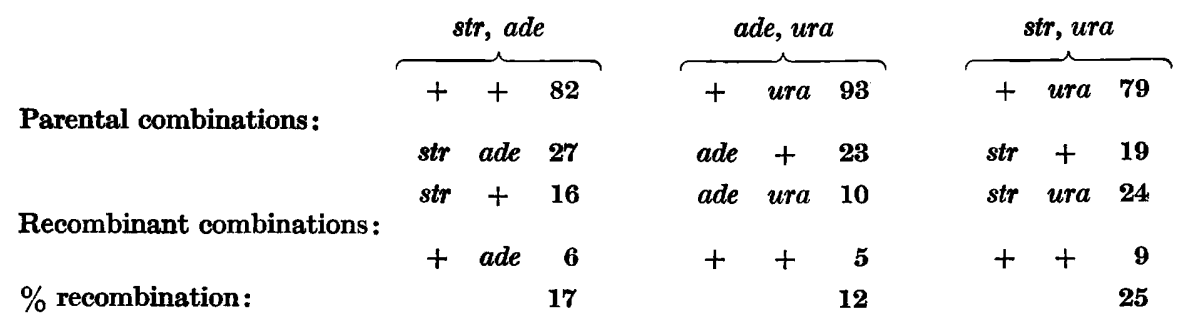

* The dotted line indicates a deleted region.

In this segregation, inequalities in the frequencies of complementary genotypes are explained by a single terminal deletion (contrast Table 3$): \chi^{2}(3$ D.F.) testing deviation from equality of ratios within the four pairs of complementary genotypes $=0.79(P=0.85)$.

Table 2 shows the data for the segregation of three loci in linkage group II from another heteroclone. Complementary genotypes do not have equal frequencies, genotypes including the allele ura-1+ always being less frequent. The diminished frequencies of the markers in coupling with ura-1+, namely ade-3- and $s t r-1^{-}$, can be attributed to the primary disturbance at the ura-1 locus; they do not require independent explanations. This is shown by the fact that none of the ratios between 
the frequencies of complementary genotypes differs significantly from the allele ratio at the ura-1 locus, that is 103:28.

Other heteroclones were found in which the magnitude of the disturbance in the allele ratio at the ura-1 locus varied widely from one heteroclone to another, one allele being occasionally completely absent. Sometimes $u r a-1^{+}$and sometimes ura-1- was the less frequent. Various other loci were found to show primary disturbances in allele ratios in different heteroclones, and like ura- 1 these were always terminal loci of one or other linkage group. The simplest explanation of these findings (Hopwood, Mancinelli, Sermonti \& Spada-Sermonti, 1961) is that the units from which the heteroclones arise are 'heterogenotes ' (Morse, Lederberg \& Lederberg, $1956 b$ ) in which one or more terminal segments of chromosome are lacking. For an allele in coupling with a deletion to be inherited by viable segregants, a crossover must occur between the locus and the point of deletion, assuming the deficiency to be lethal in the haploid condition; this accounts for the decreased frequency of the allele.

Table 3. Segregation of markers in linkage group II in a heteroclone

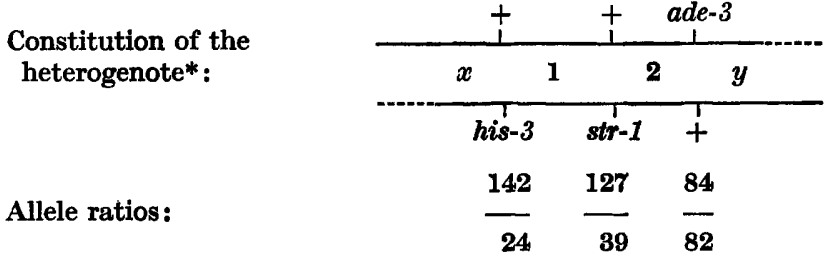

(a) Numbers of segregants of each genotype

$\begin{array}{ccc}\text { Genotypes of } & \begin{array}{c}\text { Observed } \\ \text { numbers }\end{array} & \begin{array}{c}\text { Cross-over } \\ \text { in regions }\end{array} \\ +\quad+\text { ade } & 80 & y \\ \text { his str }+ & 20 & x \\ + \text { str }+ & 18 & 1 \\ \text { his }+ \text { ade } & 3 & x, 1, y \\ +\quad+\quad+ & 43 & 2 \\ \text { his str ade } & 0 & x, 2, y \\ + \text { str ade } & 1 & 1,2, y \\ \text { his }+\quad+ & 1 & x, 1,2\end{array}$

(b) Recombination between pairs of loci

\begin{tabular}{|c|c|c|c|c|c|c|c|c|c|}
\hline \multirow[b]{2}{*}{ Parental combinations: } & \multicolumn{3}{|c|}{ str, ade } & \multicolumn{3}{|c|}{ his, str } & \multicolumn{3}{|c|}{ his, ade } \\
\hline & $\begin{array}{c}+ \\
\text { str }\end{array}$ & $\begin{array}{c}\text { ade } \\
+\end{array}$ & $\begin{array}{l}83 \\
38\end{array}$ & $\stackrel{+}{\text { his }}$ & $\begin{array}{l}+ \\
\text { str }\end{array}$ & $\begin{array}{r}123 \\
20\end{array}$ & $\stackrel{+}{\text { his }}$ & $\begin{array}{c}\text { ade } \\
+\end{array}$ & $\begin{array}{l}81 \\
21\end{array}$ \\
\hline Recombinant combinations : & $\begin{array}{l}+ \\
s t r\end{array}$ & $\underset{a d e}{+}$ & $\begin{array}{r}44 \\
1\end{array}$ & $\underset{h i s}{+}$ & $\begin{array}{c}s t r \\
+\end{array}$ & $\begin{array}{r}19 \\
4\end{array}$ & $\stackrel{+}{\text { his }}$ & $\begin{array}{c}+ \\
\text { ade }\end{array}$ & $\begin{array}{r}61 \\
3\end{array}$ \\
\hline Total recombinants: & & & 45 & & & 23 & & & 64 \\
\hline $\begin{array}{l}\% \text { recombination } \\
(\text { str-ade }=14)\end{array}$ & & & (14) & & & 7 & & & 20 \\
\hline
\end{tabular}

* Dotted lines indicate deleted regions.

In this segregation, disturbances are explained by two terminal deletions in trans (contrast Table 2): $\chi^{\mathbf{2}}(\mathbf{3}$ D.F.) testing deviation from equality of ratios within the four pairs of complementary genotypes $=17 \cdot 10(P \ll 0 \cdot 01)$. The data come from Table 1 , the locus phe-1, closely linked to ade-3, being disregarded. 
When, as in the heteroclone in Table 2, the departure from equality of complementary genotypes is attributable to a single disturbance (a single deficiency on the hypothesis of terminal deletions), the estimation of linkage between pairs of loci presents no difficulty (Table $2 b$ ). The observed proportion of segregants showing the recombinant genotypes can be taken as a reliable estimate of recombination, since the decreased frequency of a single marker affects proportionately the frequencies of parental and recombinant segregants. This situation can be recognized by means of a $2 \times 2 \chi^{2}$ which compares the ratios within parental and recombinant classes. When this chi-square is not significant, as for the three pairs of loci in Table $2 b$, the linkage estimates can be accepted as reliable to the degree of accuracy with which we are concerned here. Several pairs of markers were studied in this way in many heteroclones and, when estimates of recombination were accepted only from data giving a non-significant chi-square, an entirely consistent picture of the linkage relations of the markers resulted (Hopwood \& Sermonti, 1962). Moreover, the linkage map of Hopwood (1959), which was based on the much less direct method of selective analysis, was very largely confirmed.

Often a single disturbance will not explain the irregularities in the segregation of the markers in a linkage group. An example involving three loci in linkage group II is presented in Table 3 . As before, the frequencies of complementary genotypes are different, but this time the ratio is not the same in all pairs of genotypes. Two disturbances have to be postulated, and they can be represented as deletions at opposite ends of the two homologous chromosomes (that is in the trans configuration). The situation is most clearly illustrated if we consider the segregation of the alleles at two loci. For example, considering only the loci str-1 and ade-3, a model for this heterogenote is as follows:

\begin{tabular}{|c|c|c|}
\hline$x$ & $\mathbf{2}$ & $y$ \\
\hline
\end{tabular}

The four classes of segregants would be produced as follows:

\begin{tabular}{|c|c|c|c|}
\hline & & $\begin{array}{l}\text { Cross-over } \\
\text { in }\end{array}$ & $\begin{array}{c}\text { Observed } \\
\text { no. }\end{array}$ \\
\hline Parental classes: & $\begin{array}{c}+\quad \text { ade } \\
\text { str }\end{array}$ & $\begin{array}{l}y \\
x\end{array}$ & $\begin{array}{l}88 \\
38\end{array}$ \\
\hline Recombinant classes: & $\underset{\text { str }}{+} \underset{\text { ade }}{+}$ & $\begin{array}{c}2 \\
x, 2, y\end{array}$ & $\begin{array}{r}44 \\
1\end{array}$ \\
\hline
\end{tabular}

The greater inequality in the frequencies of the two recombinant classes as compared with the parental pair is explained by the necessity of a triple cross-over for the formation of the type str ade, as compared with single cross-overs for the other three classes. Moreover, the observed proportion of recombinants between the two loci, 45/166, is an overestimate of the true frequency of recombination. This is because the need for a cross-over in either interval $x$ or $y$ to produce the parental classes diminishes the frequencies of both of these classes, while only one of the recombinant classes suffers a decrease in frequency, this time a compound one caused by the double cross-over in $x$ and $y$; the net result of a reduction of each of the parental classes is proportionately greater than that of a compound reduction of only one of the recombinant classes. 
Even in the presence of two disturbances in one linkage group, estimates of recombination can be made. The situation of a pair of loci included between two deletions in trans is formally equivalent to the four-point bacterial cross with selection for wild-type alleles in trans at the two outside loci considered by Bailey (1951), and so on for larger numbers of loci. As pointed out by Bailey, a method of calculating recombination percentages which uses the frequencies of the classes requiring multiple cross-overs suffers from the severe drawback that these values are very small and easily biased. The safest approach is to calculate unknown recombination values in proportion to another distance in the same linkage group that is already known (from the analysis of other heteroclones). When, for example, we take the distance between $s t r-1$ and $a d e-3$ to be 14 units (the mean value from the data in Hopwood \& Sermonti, 1962), the other two recombination percentages in the heteroclone of Table 3 can be calculated by simple proportionality. The estimates obtained in this way are only first approximations, since no account is taken of multiple crossing-over, and a more complex mathematical treatment is needed to obtain more precise values. However, the correction is negligible when the various intervals are small and of comparable length.

We have seen that the data from heteroclones in which the segregation of markers in a linkage group is affected by a single disturbance or by two disturbances in the trans configuration can be used to estimate the recombination percentages between pairs of loci, and the various distances show reasonable additivity. The order of the loci on the linkage map can also be readily determined by considering the segregation of three loci, and identifying the least frequent complementary pair as that produced by double cross-over. Thus the data in Table 2 indicate the order str-1ade-3-ura-1 for these three loci, while those in Table 3 give the order his-3-str-1ade-3; these combine to give the following order for the four loci: his-3-str-1ade-3-ura-1. Many more data are summarized by Hopwood \& Sermonti (1962).

\section{The range of heteroclone types}

The heteroclones just considered were recovered on unsupplemented minimal medium, on which a number of wild-type alleles were selected, besides those at the two closely linked loci which are the minimum requirement for the recovery of heteroclones. When each parent strain carries selected markers in each linkage group, the heteroclones are normally heterozygous for markers in both linkage groups. They always show terminal deletions in at least one member of both chromosome pairs. Both linkage groups have shown the same kinds of deletions, and no relationship has been found between the deletions in the two linkage groups. Single deletions (Table 2) and double deletions at opposite ends of the two homologous chromosomes (that is in the trans configuration; Table 3) are common. Occasionally, the allele ratios suggest two deficiencies at opposite ends of the linkage group, but affecting the markers contributed by the same parent (that is in the cis configuration). However, in such cases it turns out that there has been a change in the coupling of the markers, such that the two chromosomes in the plating unit that gave rise to the heteroclone must have been non-parental. This means that the two deficiencies are on different chromosomes, so that the deletions are in the trans configuration. In some heteroclones, one allele at a terminal locus is absent. Sometimes this can be explained by the inclusion of this allele in a deletion, because the segregation of 
the other markers shows an appropriate disturbance. Sometimes, however, a terminal marker is missing, but the segregation of the other markers shows no disturbance attributable to a deletion including the missing allele. The simplest explanation is that the lack of one allele in such heteroclones is due to homozygosity, rather than hemizygosity, of the homologous allele.

When various supplements are added to the medium on which the heteroclones are recovered, so that the number of selected markers is reduced, a much wider range of heteroclone types is possible and a great variety of segregation patterns has been found amongst the several hundred heteroclones that have been studied. When the selective conditions are relaxed in this way, many heteroclones show an excess of one or two genotypes. The genotype or genotypes in excess are usually able to grow on the selective medium, and their presence is probably due to the origin, within the growing heteroclone, of recombinants which then multiply clonally. Heteroclones showing this kind of bias are the more common the fewer are the selected markers, when the probability of cross-over events that can give rise to recombinants capable of growth on the selective medium is greater. When the alleles of at least one locus in each linkage group are heterozygous, heteroclones of this kind can be recognized by the test of independence between the two linkage groups (see Table 1), which turns out to be significant.

When the plating medium is selective for heterozygosity of the markers of only one linkage group, the other linkage group is very often represented by a single set of markers, usually those of one of the parents, but occasionally a recombinant set. It seems probable that the plating units which give rise to heteroclones of this type contained an aneuploid nucleus, in which one linkage group was present in the diploid condition, and the other in the haploid condition. However, we cannot exclude the possibility that the markers of the second linkage group are also present in the diploid condition, but homozygous.

A special kind of heteroclone, which gives a highly significant independence $\chi^{2}$, is made up almost exclusively of the two parental genotypes, sometimes together with rare recombinants. These colonies are interpreted as heterokaryons, and they are particularly numerous in certain crosses.

\section{Higher-order heteroclones}

Second-order heteroclones are obtained by sowing spores from a heteroclone on a medium selective for closely linked markers in repulsion. They are much more numerous when the inoculum consists of fragments of the mycelium of the original heteroclone, instead of spores. A single colony can give rise to several thousand second-order heteroclones. The few second-order heteroclones that have so far been analysed have often shown a pattern of segregation resembling that of the parent heteroclone. However, a high proportion of them have shown greater deficiencies of markers, when the selective medium has allowed the recovery of incomplete heteroclones.

The classification of the heteroclones as first, second, or higher order merely indicates the number of transfers on selective media that have preceded their isolation. The first-order heteroclones are not necessarily derived from plating-units containing primary zygote nuclei, since their nuclei may already have undergone several multiplications in the mixed culture. The problem of isolating the zygotes 
has not yet been resolved, and so we cannot say whether they are complete or incomplete. However, comparison of the segregation patterns of first and secondorder heteroclones clearly indicates the occurrence of post-zygotic losses of markers.

\section{DISCUSSION}

From a mixed culture of two suitably marked strains of Streptomyces coelicolor strain A3(2) it was possible to isolate heterozygotes containing the marker alleles of both parents. These heterozygotes gave rise to mixed clones, the heteroclones, in which the parental and numerous recombinant genotypes were present. Therefore the heterozygotes represent a stage in the pathway of production of recombinants. We cannot, however, exclude the existence of alternative pathways of recombinant formation. The variety of recombinant genotypes recoverable from a single heteroclone is large, probably being limited only by the number of markers and the size of the sample of segregants analysed. This indicates that the number of independent events giving rise to recombinants, and so the number of heterozygous nuclei undergoing segregation in any heteroclone, is large, probably several thousand. This contrasts with the normal situation in Escherichia coli (Lederberg, 1957; Anderson, 1958), where a single zygote produced not more than four recombinant genotypes (omitting the characters of motility and morphology).

The occurrence of numerous segregational events need not necessarily imply that the heterozygous nucleus in the heteroclone multiplies equationally for many generations. Another possibility, suggested by Lederberg (1957) to account for the presence of several recombinant genotypes amongst the progeny of a single zygote of Escherichia coli is a 'recycling of meiosis', that is an alternation of haploid and diploid nuclear generations. This hypothesis has also been used to explain recombination in bacteriophage (Visconti \& Delbrück, 1953). Clearly such a process would lead to an increase in apparent recombination frequencies, which would approach $50 \%$ if the process were repeated many times. However, the two linkage groups of Streptomyces coelicolor turn out to be particularly short (Hopwood \& Sermonti, 1962), and this is not due to parental contamination, because the two linkage groups always segregate independently. Within the two linkage groups, the original coupling of the markers is preserved, with rare exceptions, in all heteroclones analysed quantitatively, whether first or higher order. Thus an extensive recycling of meiosis seems improbable.

Anderson (1958) put forward another hypothesis to account for the occurrence of repeated segregational events in the descendents of the zygotes of Escherichia coli, without equational divisions of the zygote nucleus. He suggested that the fragment of donor chromosome did not multiply, but was inherited unilinearly, segregants being produced at intervals by a copy-choice mechanism. A consequence of this hypothesis is that not more than one heterogenote could occur amongst the descendents of a single zygote; this is certainly not true in the heteroclones of Streptomyces coelicolor, each of which can give rise to several thousand segregating subclones.

These considerations suggest that the heterozygous nucleus multiplies for a number of generations, probably at least a dozen, during the development of the heteroclones of Streptomyces coelicolor. At sporulation, nearly all the spores in the heteroclone contain haploid nuclei. Crossing-over has taken place, but we cannot yet say whether this occurs only at the time of reduction to haploidy, or during the 
equational divisions of the heterozygous nuclei, nor whether it occurs simultaneously in the two linkage groups. Heteroclones heterozygous for markers in one linkage group and with the other represented by a single set of markers are very common, the latter occasionally representing a non-parental arrangement, but these markers could be present in the homozygous or hemizygous condition.

Complementary segregant classes always have different frequencies in the heteroclones, even when the markers of a single linkage group are considered. These disturbances can be attributed to a factor that reduces the frequency of one of the terminal alleles of a chromosome, or sometimes to two factors that affect two alleles at opposite ends of the two homologous chromosomes. From the point of view of the formal genetic analysis, the nature of these factors is unimportant. However, the hypothesis that they are haplo-lethal chromosome deletions appears to be the most reasonable, especially in view of the variety of deficiencies observed in different heteroclones from the same cross and in higher-order heteroclones derived from the same heterogenotic nucleus. The fact that the alleles with the lowest frequencies (or absent altogether) are always terminal ones is in favour of the hypothesis of terminal deletions.

Some rearrangements do occur during the equational divisions of the heterozygous nuclei, as shown by occasional homozygosity, and rare changes in coupling of the markers. These rearrangements could arise by mitotic crossing-over at a four-strand stage, and this could also account for the disappearance of terminal deletions and for the transfer of a deletion from one chromosome to its homologue, phenomena that are observed when certain higher-order heteroclones are compared with the parent heteroclone. The extent of these phenomena has still to be determined.

The incompleteness of the heterogenotes from which the heteroclones originate appears to be due largely to post-zygotic changes, since higher-order heteroclones show more extensive deficiencies than the heteroclones from which they originated. Completeness or incompleteness of the primary zygotes has still to be determined. Study of the heteroclones has thrown little light on the early stages of sexual reproduction, that is conjugation and the transfer of genetic material, apart from confirming the conclusion from the selective analysis of recombination that large parts of the parental genomes, if not whole genomes, must be transferred(Hopwood, 1959).

The heteroclones show striking similarities to the semi-stable heterozygous strains of Escherichia coli $\mathrm{K12}$ isolated by Lederberg (1949). These, like the heteroclones of Streptomyces coelicolor, were derived from heterogenotes whose nuclei divided equationally for a certain number of generations before segregating haploid products, they regularly lacked one or more chromosome segments derived from one or other of the parents, or occasionally from both, and they sometimes showed homozygosity of certain markers (Lederberg, Lederberg, Zinder \&Lively, 1951 ; Nelson \& Lederberg 1954). Unfortunately the heterozygotes of $\boldsymbol{E}$. coli have not been fully exploited. They were used as an argument in support of the theory of post-zygotic elimination of chromosome segments in $E$. coli (Nelson \& Lederberg, 1954), and when pre-zygotic elimination was elegantly demonstrated (Wollman, Jacob \& Hayes, 1956) they appear to have been abandoned, and have even been considered to be an example of genetic teratology (Jacob \& Wollman, 1961). However, pre-zygotic and post-zygotic elimination are not mutually exclusive; on the contrary, they may be different expressions of the same tendency towards fragmentation of the chromosome that 
seems to be a general feature of all bacterial genetic systems. The heteroclones of Streptomyces coelicolor should help to throw more light on this aspect of microbial genetics.

This work was aided by travel grants from the Italian Comitato Nazionale per l'Energia Nucleare (C.N.E.N.).

\section{REFERENCES}

Anderson, T. F. (1958). Recombination and segregation in Escherichia coli. Cold Spr. Harb. Symp. quant. Biol. 23, 47.

Baruey, N. T. J. (1951). The estimation of linkage in bacteria. Heredity, 5, 111.

Ephrussi-Taylor, H. (1960). On the biological functions of deoxyribonucleic acid. Symp. Soc. gen. Microbiol. $10,132$.

Hartman, P. E. \& GoodgaI, S. H. (1959). Bacterial genetics (with particular reference to genetic transfer). Annu. Rev. Microbiol. 13, 465.

Hayes, W. (1960). The bacterial chromosome. Symp. Soc. gen. Microbiol 10, 12.

Hopwood, D. A. (1957). Genetic recombination in Streptomyces coelicolor. J. gen. Microbiol. 16, ii.

Hopwood, D. A. (1959). Linkage and the mechanism of recombination in Streptomyces coelicolor. Ann. N.Y. Acad. Sci. 81, 887.

Hopwoon, D. A. (1960). Phase-contrast observations on Streptomyces coelicolor. J. gen. Microbiol. 22, 295.

Hopwood, D. A., Mancinelut, A., Sermonti, G. \& Spada-Sermontr, I. (1961). Eterocloni in Streptomyces. Atti Assoc. Genet. Ital. 6, 71.

Hopwood, D. A. \& Sermontr, G. (1962). The genetics of Streptomyces coelicolor. Advanc. Genet. 11, 273.

JACOB, F. \& ADELBerg, E. A. (1959). Transfert de caractères génétiques par incorporation au facteur sexuel d'Escherichia coli. C.R. Acad. Sci., Paris, 249, 189.

JACOB, F. \& Wollman, E. L. (1958). Les èpisomes, éléments génétiques ajoutés. C.R. Acad. Sci., Paris, 247, 154.

JACOB, F. \& Wollman, E. L. (1961). Sexuality and the Genetics of Bacteria. New York: Academic Press.

Lederberg, J. (1949). Aberrant heterozygotes in Escherichia coli. Proc. nat. Acad. Sci., Wash. 35, 178.

Lederberg, J. (1957). Sibling recombinants in zygote pedigrees of Escherichia coli. Proc. nat. Acad. Sci., Wash. 43, 1060.

LEDERBERG, J. \& LeDERBERG, E. M. (1952). Replica plating and indirect selection of bacterial mutants. J. Bact. 63, 399.

LederberG, J., Lederberg, E. M., Zinder, N. D. \& Lively, E. R. (1951). Recombinational analysis of bacterial heredity. Cold Spr. Harb. Symp. quant. Biol. 16, 413.

Morse, M. L., Lederberg, E. M. \& Lederberg, J. (1956a). Transduction in Escherichia coli K12. Genetics, 41, 142.

Morse, M. L., Lederberg, E. M. \& Lederberg, J. (1956b). Transductional heterogenotes in Escherichia coli. Genetics, 41, 758.

Nelson, T. C. \& Lederberg, J. (1954). Postzygotic elimination of genetic factors in Escherichia coli. Proc. nat. Acad. Sci., Wash. 40, 415.

Sermonti, G. \& Spada-Sermontr, I. (1955). Genetic recombination in Streptomyces. Nature, Lond. 176, 121.

Sermonti, G., Mancinelit, A. \& Spada-Sermonti, I. (1960). Heterogeneous clones (heteroclones) in Streptomyces coelicolor A3(2). Genetics, 45, 669.

Visconti, N. \& Delbrück, M. (1953). The mechanism of genetic recombination in phage. Genetics, 38, 5.

Wollman, E. L., JACOB, F. \& HAYes, W. (1956). Conjugation and genetic recombination in Escherichia coli. Cold Spr. Harb. Symp. quant. Biol. 21, 141.

WoolF, B. (1957). The log likelihood ratio test (the $G$-test): methods and tables for tests of heterogeneity in contingency tables. Ann. Human. Genet., Lond. 21, 397. 